\title{
Emerging Beam Resonances in Atom Diffraction from a Reflection Grating
}

\author{
Bum Suk Zhao (조범석), ${ }^{*}$ Gerard Meijer, and Wieland Schöllkopf ${ }^{\dagger}$ \\ Fritz-Haber-Institut der Max-Planck-Gesellschaft, Faradayweg 4-6, 14195 Berlin, Germany
}

(Received 18 February 2010; published 16 June 2010)

\begin{abstract}
We report on the observation of emerging beam resonances, well known as Rayleigh-Wood anomalies and threshold resonances in photon and electron diffraction, respectively, in an atom-optical diffraction experiment. Diffraction of He atom beams reflected from a blazed ruled grating at grazing incidence has been investigated. The total reflectivity of the grating as well as the intensities of the diffracted beams reveal anomalies at the Rayleigh angles of incidence, i.e., when another diffracted beam emerges parallel to the grating surface. The observed anomalies are discussed in terms of the classical wave-optical model of Rayleigh and Fano.
\end{abstract}

DOI: 10.1103/PhysRevLett.104.240404

It is a general phenomenon in wave optics that diffraction by a periodic surface shows peculiar intensity variations in the outgoing beams when conditions (i.e., wavelength, periodicity, and incidence angle) are such that a diffracted beam just emerges parallel to the surface. This was first observed by Wood in 1902 [1] who found strange dark and bright bands (Wood anomalies) in diffraction patterns of white light from ruled reflection gratings. Rayleigh in 1907 first traced back Wood anomalies to grazing emergence of a diffracted beam [2]. Thus, the term Rayleigh conditions (Rayleigh wavelength, Rayleigh angle) refers to conditions for grazing beam emergence where Wood anomalies occur. Rayleigh and subsequently Fano explained the anomalous behavior by interference between first and second order scattering of the incident wave, where second order scattering refers to waves that are first scattered from a neighboring grating stripe [2-4]. Later on Wood anomalies were categorized into two cases, one (sharp anomalies) related exclusively to the emergence of a diffracted beam (Rayleigh-Wood anomaly) and the other (broad anomalies) to resonance effects $[4,5]$. The resonance type Wood anomaly is attributed to excitation of surface-plasma oscillations guided along the grating surface [6,7]. In recent years the effect of extraordinary optical transmission through periodic arrays of subwavelength holes [8] has been explained in terms of Wood anomalies $[9,10]$. In addition, Rayleigh-Wood anomalies have been observed in soft x-ray diffraction [11] and have been considered in designing x-ray monochromators [12].

The effect has also been studied independently in matter-wave optics such as reflection high energy electron diffraction, low energy electron diffraction, and atom and molecular beam scattering from crystal surfaces [13]. In low energy electron diffraction, for example, it was first observed in unfocused electron beam diffraction from crystal surfaces $[14,15]$. Here, a crystal surface was used instead of a grating surface, since a periodic length on the order of an $\AA$ is required for the diffraction of the electron due to its small de Broglie wavelength. The parlance used in electron diffraction is, however, different from classical
PACS numbers: 03.75.Be, 37.25.+k, 42.25.Fx, 68.49.Bc

optics using the terms threshold effect and electronic surface resonance instead of the counterparts in optics, Rayleigh-Wood and resonance type anomaly, respectively [13]. Also, the emerging beam condition given by the Rayleigh wavelength was referred to as type $K_{\text {II }}$ Kikuchi lines [16].

In atom optics a behavior in analogy to the resonance type anomaly and the electronic surface resonance has been investigated for a long time under the name of selective adsorption [17]. Estermann and Stern in 1930 observed anomalous intensity fluctuations in the specular peak of helium diffraction from a crystal surface [18]. The anomaly was accounted for by a bound state of the atomsurface interaction potential [19]. The relation between selective adsorption and Wood anomaly was discussed by Fano already in 1938 [3]. On the other hand, RayleighWood anomalies have not been observed in atom-surface scattering experiments, although they have been predicted by theory [20-22]. In these theoretical studies the anomaly was referred to as threshold resonance or emerging beam resonance. As the latter term is more descriptive, it is adopted here. More recently, Guantes et al. suggested that emerging beam resonances should be observable in elastic atom scattering from a highly corrugated surface [23].

Here we report the first observation of emerging beam resonances in an atom-surface scattering experiment, complementing Rayleigh-Wood anomalies observed with light and electrons. Helium atom beams are diffracted from a plane ruled grating at near grazing incidence. By varying the incidence angle we observe the resonances precisely at the Rayleigh incidence angles in two ways: (i) the total coherent reflectivity of the grating increases steeply, (ii) the intensity curves of the diffracted beams and the specular beam exhibit abrupt changes of their slopes. We adopt the multiple scattering approach of Rayleigh [2] and Fano $[3,4]$ to explain basic features of our observations.

As predicted by Armand and Manson [22] emerging beam resonances in atom-surface scattering occur within a small angular range. Thus, the collimation of the incident 
beam and the angular resolution of detection are required to be on the order of $100 \mu \mathrm{rad}$. Another prerequisite for observing the effect is to have a significant flux diffracted into the very beam that, at Rayleigh conditions, emerges above the surface so as to have an appreciable effect on the other outgoing beam intensities. In the experiment described here these requirements have been met by reflecting a highly collimated helium atom beam at grazing incidence from a blazed ruled diffraction grating. The use of helium atoms at grazing incidence ensures sufficient coherent reflection probability [24], while the grating blaze angle in combination with a well chosen azimuthal orientation of the grating leads to an effective enhancement of the intensity of the emerging diffracted beam.

The high angular resolution diffraction apparatus has been used in previous experiments [24,25]. The continuous atom beam is formed by supersonic expansion of $\mathrm{He}$ gas at stagnation temperature $T_{0}=8.7 \mathrm{~K}$ and pressure $P_{0}=0.5$ bar through a 5 - $\mu \mathrm{m}$-diameter orifice into high vacuum. After passing a skimmer of $500 \mu \mathrm{m}$ diameter, the beam is collimated by two $20 \mu \mathrm{m}$ wide slits (slit 1 and slit 2) separated by $100 \mathrm{~cm}$ as indicated in Fig. 1. In combination with the $25 \mu \mathrm{m}$ wide detector-entrance slit (slit 3), located $78 \mathrm{~cm}$ downstream from the second slit, the observed angular width of the atom beam is $120 \mu \mathrm{rad}$ (full width at half maximum). The third slit and the detector (an electron-impact ionization mass spectrometer) are mounted on a frame which is rotated precisely as indicated
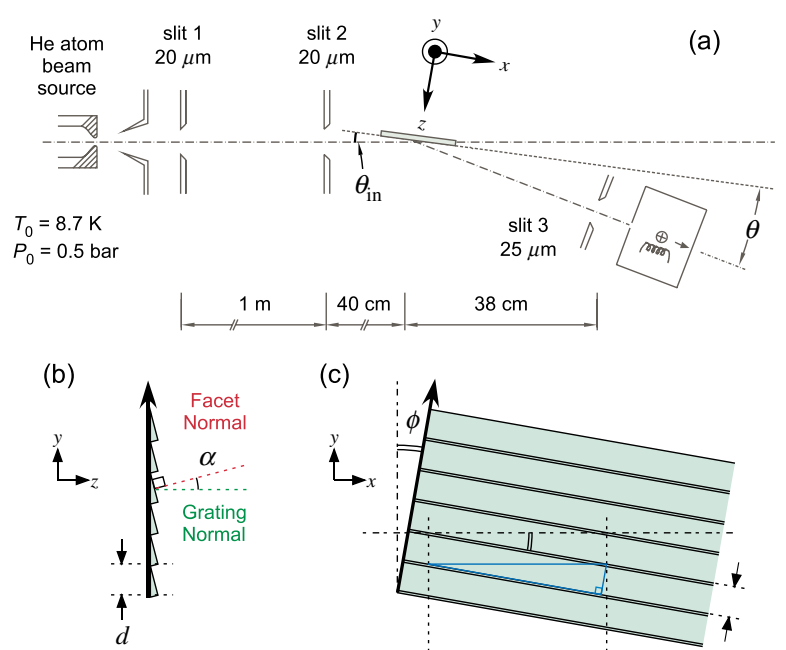

(d)

(c)

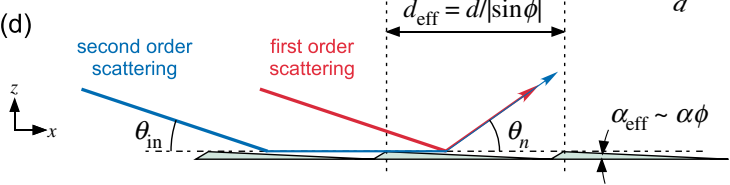

FIG. 1 (color online). Scheme of the experimental setup and orientation of the plane ruled grating. In each figure the chosen coordinate system is denoted. The grating azimuth angle $\phi$ is the angle between the grating blaze arrow [thick arrow in (b) and (c)] and the $y$ axis. In (b) $\phi=0$, whereas (c) and (d) correspond to $\phi<0$. in Fig. 1(a). A plane ruled grating is positioned such that the detector pivot axis is parallel to the grating surface and passes through its center. The pivot axis (vertical), and the grating normal (horizontal), are chosen as the $y$ and $z$ axis of the reference frame, respectively. Hence, the (horizontal) $x z$ plane is the plane of incidence. The grazing incidence angle $\theta_{\text {in }}$ and the detection angle $\theta$ are measured with respect to the grating surface plane. Diffraction patterns are obtained by rotating the detector, namely, varying $\theta$, and measuring the He signal at each angle.

The commercial plane ruled grating (Newport 20RG050-600-1) is made out of $6 \mathrm{~mm}$ thick glass with an aluminum coating and has a surface area of $5 \times 5 \mathrm{~cm}^{2}$. It is characterized by a period $d=20 \mu \mathrm{m}$ and a blaze angle $\alpha=0.8^{\circ}(\approx 14 \mathrm{mrad})$ [see Fig. 1(b)]. The orientation of the grating in space is defined by the grating normal and the blaze arrow. The latter is perpendicular to the grating normal and to the grating grooves and points vertically upward in Fig. 1(b).

The grating is aligned such that its grooves are almost (but not quite) parallel to the $x$ axis (horizontal). This geometry corresponds to the conical diffraction mode known from extreme ultraviolet spectroscopy [26]. In this geometry out-of-plane diffraction is without effect on the measurements because the vertical acceptance angle of slit $3(\approx 10 \mathrm{mrad})$ is far larger than the vertical diffraction angles (tens of $\mu \mathrm{rad}$ ). As indicated in Fig. 1(c), the grating can be rotated by the azimuth angle $\phi$ around the $z$ axis. By varying $\phi$ the effective periodic length $d_{\text {eff }}$ and the effective blaze angle $\alpha_{\text {eff }}$ can be adjusted, as depicted in Figs. 1(c) and 1(d). The former is given by trigonometry of the triangle in Fig. 1(c), $d_{\text {eff }}=d /|\sin \phi|$, while the latter is approximated by $\alpha_{\text {eff }} \approx \alpha \phi$, which is derived from $\sin \alpha_{\text {eff }}=\sin \alpha \sin \phi$. We define $\phi$ to be negative (positive) when the blaze arrow is rotated clockwise (counterclockwise) from the $y$ axis. In this convention Fig. 1(c) shows the case of $\phi<0$. Since the effect of blazing is to enhance those diffracted beams which are specularly reflected with respect to the facet normal, negative (positive) diffraction orders get enhanced for $\phi<0(\phi>0)$.

The fraction of He atoms that are coherently scattered from the grating, measured for three different azimuth angles $\phi<0$, is plotted as a function of incidence angle in Fig. 2. It is determined from the summation of the areas $A_{n}$ of the diffraction peaks in intensity vs detection angle plots (see below). The sum is taken over all diffraction orders $n$ (including $n=0$, the specular peak) and normalized to the incidence beam area $A_{\text {in }}$. The latter is measured when the grating is moved out of the beam path. The vertical lines in Fig. 2, each labeled by an integer, indicate the positions of the Rayleigh angles of incidence $\theta_{R, m}$ with the integer indicating the diffraction order $m$ of the emerging beam. The Rayleigh angle of incidence is calculated from the grating equation, $\cos \theta_{R, m}-1=m \frac{\lambda}{d_{\text {eff }}}$ [27]. $(\lambda=$ $3.32 \AA$ is the de Broglie wavelength of the helium atoms at $T_{0}=8.7 \mathrm{~K}$.) 


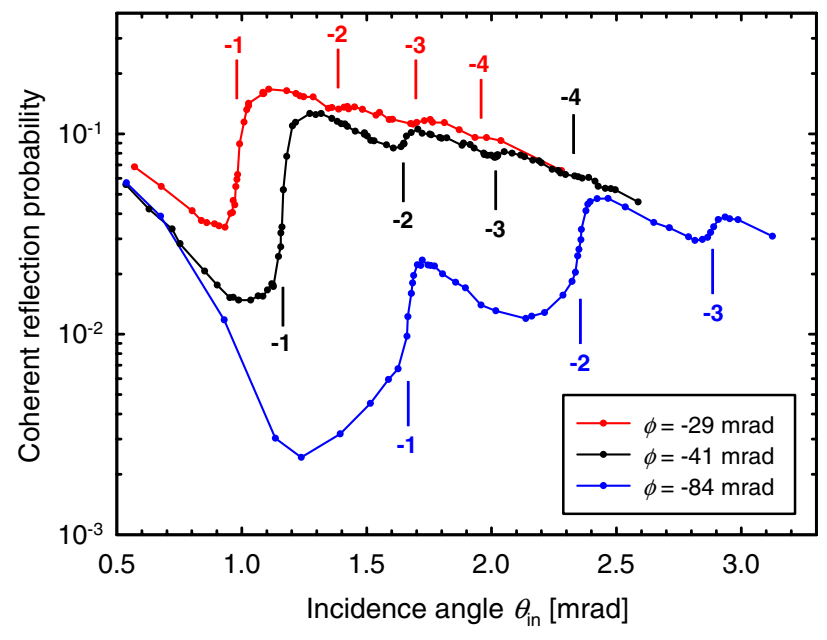

FIG. 2 (color online). Fraction of He atoms that are coherently scattered from the plane ruled grating for different azimuth angles $\phi=-29,-41,-84 \mathrm{mrad}$. Vertical lines indicate the positions of the Rayleigh angles of incidence with the numbers indicating the diffraction order of the emerging beam. (The diffraction order sign convention follows Ref. [12].)

The coherent reflection probability curves are not monotonically decaying with incidence angle as observed in previous diffraction experiments [24]. Instead, strong and abrupt variations are found at exactly the Rayleigh angles. This indicates that emergence of another diffraction beam not only leads to a redistribution of flux among the outgoing beams, but also to an increase of the fraction of $\mathrm{He}$ atoms that are coherently scattered. There must be a concurrent decrease of the fraction of He atoms that undergo diffuse scattering at the surface.

The azimuth angle $\phi$ is obtained by analyzing diffraction patterns at various incidence angles. Figure 3(a) shows diffraction spectra for $\phi=-41 \mathrm{mrad}$ at five different incidence angles in the vicinity of the Rayleigh incidence angle $\theta_{R,-1}=1.164 \mathrm{mrad}$, where the -1 st-order peak emerges. The diffraction angles $\theta_{n}$ and areas $A_{n}$ of the $n$ th-order diffraction peak are found by fitting each peak with a Gaussian curve. The incidence angle $\theta_{\text {in }}$ is determined from the detection angle of the specular peak, $\theta_{0}$. The diffraction angles $\theta_{n}$ as a function of $\theta_{\text {in }}$ are then fitted by the grating equation, $\cos \theta_{\text {in }}-\cos \theta_{n}=n \frac{\lambda}{d_{\text {eff }}}$ with $d_{\text {eff }}$ being the only fit parameter. For the data set in Fig. $3 d_{\text {eff }}=$ $493 \pm 1 \mu \mathrm{m}$ is found corresponding to an azimuth angle $\phi=-41 \mathrm{mrad}$.

The angular spectra shown in Fig. 3(a) illustrate the progressive emergence of the -1 st-order peak. The height of the latter increases rapidly from 40 to 1050 counts/s within this range of incidence angles, whereas the height of the specular peak increases from 270 to 360 counts/s when $\theta_{\text {in }}$ is increased from 1.146 to $1.179 \mathrm{mrad}$, and stays around 360 counts $/ \mathrm{s}$ at $\theta_{\text {in }}=1.203 \mathrm{mrad}$. This indicates a discontinuity of the slope of the specular intensity variation around $\theta_{\text {in }}=1.179 \mathrm{mrad}$, which agrees with the calculated Rayleigh angle. As can be seen in Fig. 3(a), this
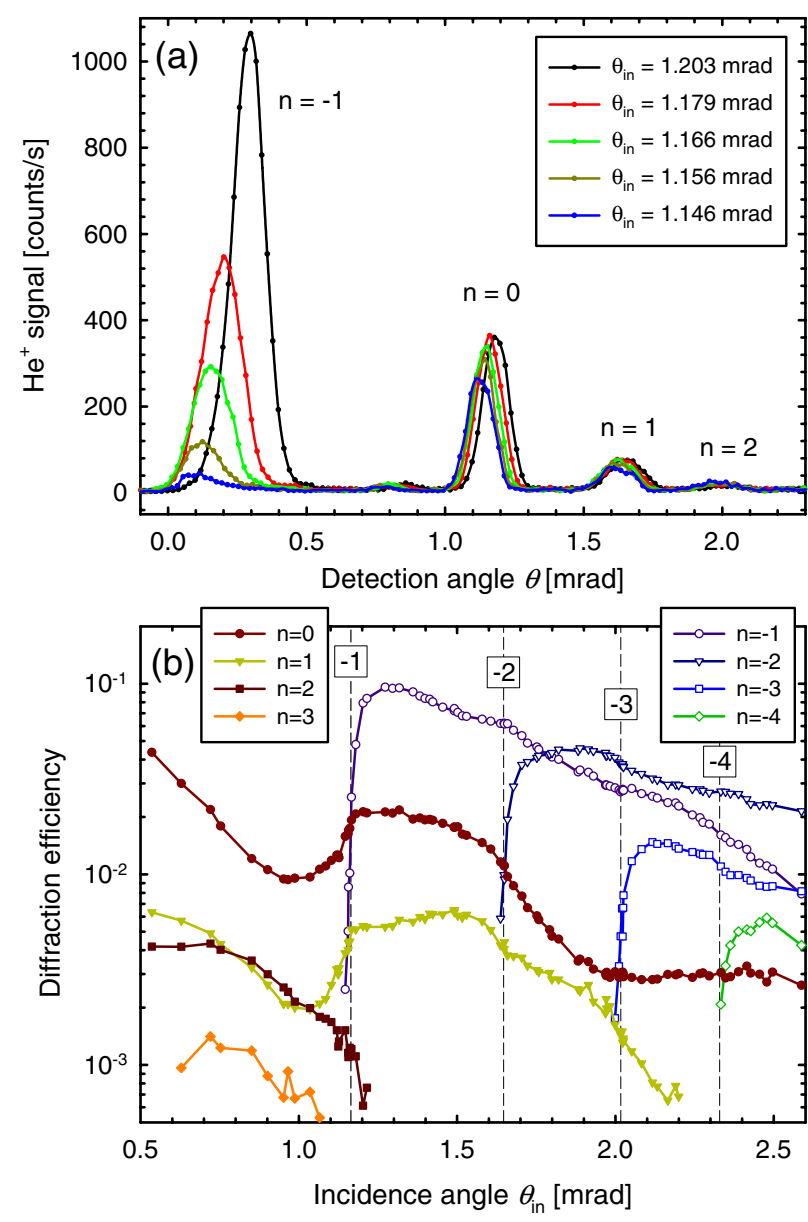

FIG. 3 (color online). (a) Diffraction patterns of $\mathrm{He}$ atom beams for $\phi=-41 \mathrm{mrad}$ for 5 different incidence angles in the vicinity of the Rayleigh incidence angle $\theta_{R,-1}=1.164 \mathrm{mrad}$ where the -1 st-order peak emerges, (b) diffraction efficiencies for $\phi=-41 \mathrm{mrad}$ as a function of incidence angle. The dashed vertical lines indicate the calculated Rayleigh incidence angles corresponding to the emergence of the $-1 \mathrm{st},-2 \mathrm{nd},-3 \mathrm{rd}$, and -4 th-order diffraction peaks.

coincides with the incidence angle at which the -1 st-order diffraction peak starts to be fully separated from the surface. The high intensity of the diffraction beam at grazing emergence is remarkable and might be a manifestation of the reciprocity theorem, as observed before in $\mathrm{x}$-ray grating diffraction [28].

Details of the emerging beam resonances can be seen more easily in the semilogarithmic plot of Fig. 3(b) showing the diffraction efficiency $A_{n} / A_{\text {in }}$ over a large range of incidence angles from 0.5 to $2.6 \mathrm{mrad}$. The different symbols correspond to various diffraction orders from $n=-4$ to 3. The dashed vertical lines indicate the Rayleigh angles of incidence. Figure 3(b) exemplifies general aspects of emerging beam resonances, which have also been found for further azimuth angles not shown here. (I) The resonance behavior at $\theta_{R, m}$ observed in the outgoing beams of order $n$ (with $n \neq m$ ) is the more distinctive, the more intense the emerging beam is. This can be seen in the figure 
where the resonance behavior is most pronounced at $\theta_{R,-1}$, less distinct at $\theta_{R-2}$, and hardly visible when the less intense -3 rd and -4 th-order diffraction peaks emerge. (II) Discontinuities seem to appear in all the other diffraction peaks, although they are most pronounced in neighboring diffraction orders, namely, $A_{m+1}$ and $A_{m+2}$. In Fig. 3(b), for instance, at $\theta_{R,-1}$ pronounced discontinuities are found in the slopes of $A_{0}$ and $A_{1}$. (III) At the Rayleigh angle of incidence the slope of $A_{n}$ usually exhibits a discontinuous decrease with increasing incidence angle, except for a few cases where a discontinuous increase is observed. In the figure, $A_{0}$ and $A_{1}$ are found to increase steeply (with the slope being close to diverging) at $\theta_{\text {in }} \leq$ $\theta_{R,-1}$, whereas they hardly change right after the vertical line. Similarly, at $\theta_{R,-2}$ the slope of $A_{-1}$ shows a sudden decrease; the slope of $A_{1}$, however, abruptly increases.

Following the approach introduced by Rayleigh and Fano [2-4] the amplitude of the $n$ th-order diffraction $S_{n}$ is approximated by interference of the amplitudes from the first and the second order scattering, $S_{n}=S_{n}^{(1)}+S_{n}^{(2)}$. The first order scattering is the scattering of the incident beam at a given grating unit [red arrow in Fig. 1(d)], while the second order scattering is the scattering of a beam at that grating unit, which has already undergone scattering at another grating unit [blue arrow in Fig. 1(d)]. When the $m$ th diffraction order fulfills the Rayleigh condition, $S_{n}^{(2)}$ is proportional to $S_{m}^{(1)}$ and the interference is constructive. Thus, the sudden increase of the emerging $m$ th-order intensity increases the other orders [aspects (II) and (III)]. The degree of the influence is proportional to $S_{m}^{(1)}$, namely, $A_{m}$ [aspect (I)]. Therefore, once the $m$ th-order peak gets separated from the grating, as seen in the spectra of Fig. 3(a), the contribution of the second order scattering to the other diffraction beams diminishes and their intensities level off.

Emerging beam resonances, i.e., Rayleigh-Wood anomalies in atom optics, have been predicted to provide detailed information of atom-surface interaction potentials [20-22]. However, more than a century after the first observation of Wood anomalies [1] and 80 years after the first observation of selective adsorption, i.e., a resonance type Wood anomaly in atom-surface scattering [18], they were still not observed in atom diffraction experiments. Here we report the first observation of emerging beam resonances in atom optics using a blazed ruled grating at grazing incidence with the grooves oriented almost parallel to the incident beam direction. The total coherent reflectivity of the grating as well as the intensities of the diffracted beams reveal anomalies at the Rayleigh angles of incidence which are interpreted with the approach developed many decades ago to describe the anomalies observed with photons $[2,4]$. Therefore, this observation completes the analogy between photon optics (Rayleigh-Wood anomaly and resonance type Wood anomaly) on the one hand and atom optics (emerging beam resonance and selective adsorption) on the other hand.

B.S.Z. acknowledges support by the Alexander von Humboldt Foundation and by the Korea Research Foundation Grant funded by the Korean Government (KRF-2005-214-C00188). We thank J. R. Manson for insightful discussions and H.C. Schewe for help with the apparatus.

*zhao@fhi-berlin.mpg.de

†wschoell@fhi-berlin.mpg.de

[1] R. W. Wood, Philos. Mag. 4, 396 (1902).

[2] Lord Rayleigh, Philos. Mag. 14, 60 (1907).

[3] U. Fano, Ann. Phys. (Leipzig) 424, 393 (1938).

[4] U. Fano, J. Opt. Soc. Am. 31, 213 (1941).

[5] A. Hessel and A. Oliner, Appl. Opt. 4, 1275 (1965).

[6] Y.-Y. Teng and E. A. Stern, Phys. Rev. Lett. 19, 511 (1967).

[7] R.H. Ritchie, E. T. Arakawa, J. J. Cowan, and R. N. Hamm, Phys. Rev. Lett. 21, 1530 (1968).

[8] T. Ebbesen, H. Lezec, H. Ghaemi, T. Thio, and P. Wolff, Nature (London) 391, 667 (1998).

[9] M. Sarrazin, J.-P. Vigneron, and J.-M. Vigoureux, Phys. Rev. B 67, 085415 (2003).

[10] F. J. García de Abajo, Rev. Mod. Phys. 79, 1267 (2007).

[11] S. Fujiwara and Y. Iguchi, J. Opt. Soc. Am. 58, 1189 (1968).

[12] H. Padmore, V. Martynov, and K. Holis, Nucl. Instrum. Methods Phys. Res., Sect. A 347, 206 (1994).

[13] E. McRae, Rev. Mod. Phys. 51, 541 (1979).

[14] E. McRae and C. Caldwell, Surf. Sci. 7, 41 (1967).

[15] E. McRae, Surf. Sci. 25, 491 (1971).

[16] The Structure and Chemistry of Solid Surfaces, edited by G. A. Somorjai (Wiley, New York, 1969).

[17] Helium Atom Scattering from Surfaces, edited by E. Hulpke (Springer, Berlin, 1992), p. 113.

[18] I. Estermann and O. Stern, Z. Phys. 61, 95 (1930).

[19] J. Lennard-Jones and A. Devonshire, Nature (London) 137, 1069 (1936).

[20] N. Cabrera and S. Solana, Proc. Int. School of Physics, Enrico Fermi (Compositori, Bologna, 1974), p. 530.

[21] N. García and W. Schlup, Surf. Sci. 122, L657 (1982).

[22] G. Armand and J. Manson, Surf. Sci. 169, 216 (1986).

[23] R. Guantes et al., Surf. Sci. 375, L379 (1997).

[24] B. S. Zhao et al., Phys. Rev. A 78, 010902(R) (2008).

[25] H. C. Schewe et al., New J. Phys. 11, 113030 (2009).

[26] W. Cash, Appl. Opt. 21, 710 (1982).

[27] For the conical diffraction geometry a modified grating equation holds, taking into account the out-of-plane (i.e., conical) diffraction [26]. However, for our conditions (i.e., $|n \lambda / d| \ll|\sin \phi|)$ the in-plane grating equation employed with $d_{\text {eff }}$ is a very good approximation.

[28] W. Jark, Nucl. Instrum. Methods Phys. Res., Sect. A 266, 414 (1988). 